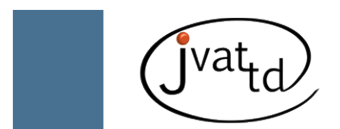

\title{
Comparison of real-time PCR and conventional PCR for detection of Leishmania (Leishmania) infantum infection: a mini-review
}

Paiva-Cavalcanti M (1), Regis-da-Silva CG (1), Gomes YM (1)

(1) Department of Immunology, Research Center Aggeu Magalhães, Oswaldo Cruz Foundation, FIOCRUZ, Recife, Pernambuco State, Brazil.

\begin{abstract}
In recent years, the polymerase chain reaction (PCR) technique has significantly advanced towards expanding its use and versatility by working with quantitative real-time PCR (qPCR). Data from the literature show that both methods present interesting characteristics for the diagnosis of visceral leishmaniasis. The benefits of qPCR in relation to conventional PCR include speed, reproducibility and quantitative ability. In addition to operational advantages, $\mathrm{QPCR}$ is more sensitive and reproducible and may replace conventional PCR in diagnostic routines. Regarding visceral leishmaniasis, the possibility of deployment of real-time PCR in highly complex diagnoses (reference services) in endemic areas will facilitate a swift and safe return for patients. Moreover, the use of a technique that possesses elevated diagnostic sensitivity, and can monitor therapy and prevent relapses promotes broader prospects for the disease control.
\end{abstract}

Key words: diagnosis, molecular diagnosis, visceral leishmaniasis.

\section{INTRODUCTION}

Visceral leishmaniasis (VL) comprises an important public health problem, since it is frequently fatal if untreated, with almost 500.000 new cases per year and more than 50.000 deaths (1). Consequently, new methods of diagnosis that promote early identification of the disease are required as well as rapid therapy.

VL diagnosis may be carried out by direct parasitological tests that can produce false-negative results, either due to the low number of Leishmania spp. organisms in clinical samples (bone marrow and lymph nodes) or because morphological identification is complex. Moreover, such methods are invasive. Conventional serological techniques are limited by cross-reactivity with other parasitic diseases and because several technical procedures have not been standardized (2). However, serological methods are still widely employed in epidemiological surveys.
Early diagnosis is important for preventing severe damage or even death of patients (3). The identification of Leishmania infections using culture or serological techniques is a prolonged process with low specificity (4). On the other hand, since the production of the first thermal cycler instrument in 1987, molecular techniques have promised a revolution in the detection of pathogens from clinical specimens (5). In this context, molecular methods, essentially based on PCR, have become indispensable tools for the diagnosis of infectious diseases (6).

High sensitivity and specificity, rapid identification of the parasite, the possibility of direct application on clinical specimens producing reliable results in a few hours are undeniable advantages of conventional PCR (cnPCR) when compared to traditional diagnosis methods of VL (parasitological and serological techniques). These advantages indicate the adoption of cnPCR in situations in which the 
conventional methods are not sufficient to reach the diagnosis of leishmaniasis in Brazil. On the other hand, in recent years, the cnPCR method has significantly advanced, working with real-time PCR or quantitative real-time PCR (qPCR). This technology promotes an accurate quantification in real-time of the amplicon, allowing the monitoring of the reaction.

Data from the world literature show that both technologies (cnPCR and qPCR) present features that are relevant for VL diagnosis.

\section{METHODS OF AMPLIFYING DNA: FEATURES}

cnPCR imitates in vitro the natural DNA replication ability, which may be repeated on a large scale. The methodology requires, first, the identification, at least in part, of the DNA target for the development of primers or probes that will hybridize specifically to the target sequence. With the increased number of sequenced pathogen genomes, catalogs of DNA sequences can be exploited for the development of diagnostic tests based on PCR, as a result, since the past decade, numerous tests commercially available are based on this technique (7).

In the past two decades, cnPCR technique has been modified to expand its use and versatility (7). The possibility of using it in the same reaction, on a pair of primers with simultaneous amplification for multiple target DNA sequences is called multiplex-PCR. Thus, more than one DNA sequence may be amplified (multiplied) in the same process (8). Nested-PCR employs two pairs of primers for amplification of an internal DNA sequence in the selected target. The first pair is used for a initial reaction, which products are then subjected to a second amplification with another pair of primers. This technique presents increased sensitivity and specificity; however, it also reveals augmented risk of contamination by the amplified product from the first reaction.

Although cnPCR and its variations are highly sensitive and specific, they have some limitations including the requirement of agarose or polyacrylamide gel for electrophoresis, risk of contamination, lack of quantitative capacity, and the use of reagents such as ethidium bromide, which is harmful to the health of the operator. The emergence of a new technology, qPCR, has emphasized such limitations.

\section{REAL-TIME PCR}

Real-time PCR was developed in 1992, as a refinement of the original PCR created by Kary Mullis, and represents a significant biotechnological breakthrough for the diagnosis of infectious and parasitic diseases (9). The system is based on the use of dyes or fluorescent probes that permit the monitoring of the amplified product. A dye widely employed is SYBR Green I, which binds nonspecifically the duplexes of DNA generated during the amplification. Another way to generate the fluorescence is to use a probe specifically targeted to a region of internal sequence that needs to be amplified, an example of this system is the TaqMan probe. During amplification, the TaqMan is degraded and there is the release of reporter that emits light $(7,10)$.

The light emission analysis of is made by a light signal detector that creates a graphic with the absorption obtained after each round of PCR, the generated signal reflects the amount of product formed (11). The cycle in which the limit of negativity or threshold exceeds is directly related to the amount of amplified DNA, and is called cycle threshold (Ct) representing the point at which the emission of fluorescence in the sample test surpasses the background (10).

The $\mathrm{qPCR}$ results are recorded through interconnected computer graphics generated in the thermal cycler. Basically, four kinds of analysis are carried out: amplification curve, dissociation curve, spectrum, and component (12).

- Through the amplification curve, the $\mathrm{Ct}$ of each sample analyzed may be checked, which is of crucial importance for the calculation of specificity $(\sigma)$ and determination of the positive samples. Values of above $35 \mathrm{Cts}$ indicate false-positive results (13).

- The dissociation curve is employed to indicate the emission of fluorescence by melting temperature (Tm). Non-specific amplification of primer dimers can be verified by this analysis.

- Spectrum assessment shows the capture of fluorescence by certain filters. SYBR Green $\mathrm{I}$, for example, is captured by filters A and B, while ROX (background ABI PRISM 7000 and $7500^{\circ}$ Applied Biosystems, USA) is read by filters $\mathrm{C}$ and $\mathrm{D}$. Thus, to the extent that a positive reaction occurs, the florescent absorption by $\mathrm{A}$ 
and B filters should be greater than that of $C$ and $\mathrm{D}$, which remains constant. If SYBR Green does not exceed ROX, the reaction is negative.

- The component shows the behavior of the fluorescence emission of each dye or reporter used during the reaction. Consequently, the background should remain constant and the employed dye should outrange it according to how many PCR cycles are being carried out. It is important to note that, if a TaqMan probe is used, three parameters are highlighted: the background, reporter, and light inhibitor signal (the quencher).

The four parameters must be considered together. Positive standards and negative controls should be included in all reactions. Optimal behavior of standard samples during the chemical reactions ensures the results of the other samples.

The qPCR allows, basically, the completion of four types of tests: absolute quantification, relative quantification, high melting resolution analysis, and allelic discrimination, which present different and varied applications (14-17). As a diagnostic tool, the absolute quantification can be used for infection detection and quantification of its etiologic agent. The test for absolute quantification is based on the standard curve analysis.

As defined, the standard curve is related to concentrations of DNA standards. Through these data, the known amount of DNA, the software quantifies the target DNA in the test sample. The standard curve also provides the slope, composed of the points on the curve. This finding is important to calculate the efficiency of amplification $(\varepsilon)$. High efficiency is associated with a slope of approximately 3.32 for each dilution of 10 of the target (13). A slope of -3.3 is connected with $100 \%$ efficiency, which indicates that the number of amplified molecules doubles with each PCR cycle (11).

These features of qPCR enable the elimination of a laborious post-amplification stage (gel electrophoresis preparation), conventionally required for the observation of the amplified product. Thus, the benefits of qPCR in relation to cnPCR are numerous and include speed, reproducibility, and quantitative ability (7).

\section{CNPCR AND QPCR FOR DIAGNOSIS OF VISCERAL LEISHMANIASIS}

In 1983, the development of cnPCR was a great progress for molecular biology (18). At that time, several researchers had begun to evaluate this technology for diagnostic purposes (19-21). Thus, the RV1 and RV2 system is known by the name of their primers, targeting $L$. infantum conserved region of $\mathrm{kDNA}$, and amplifying 145 base pair (bp) fragments (20). Initially, it revealed an excellent sensitivity in detecting L. infantum in human macrophages, about one parasite in $10^{6}$ experimentally infected mononuclear cells (20). Moreover, after a serological survey among blood donors in southeastern France, 76 positive results were obtained by Western blotting for the detection of anti-L. infantum antibodies (19). Of these, 73 buffy coats were evaluated again by cnPCR using the RV1/RV2 system, resulting in nine positive samples. Such study showed the importance of performing a survey for VL in blood donors from an endemic area, and also indicated that despite using a mononuclear cell sample for DNA extraction, the sensitivity of the RV1/RV2 system was low in comparison with the serology test.

In 2002, a work that was evaluating two methods of cnPCR found a sensitivity of $100 \%$ for the RV1/RV2 system in peripheral blood from sick dogs (22). The results encouraged the researchers to perform a study on the ability of cnPCR (RV1/ RV2) to detect VL in different samples (biopsies of the liver, spleen and lymph node) from dogs (23). The authors observed that most animals that were positive by the microscopy were also positive by PCR. Such finding could possibly be associated with the type of the sample studied or with a high parasite load in animals with classic symptoms of the disease. The sensitivity reported for the study conducted in 2002 was also determined by blood samples from dogs with multiple symptoms of VL (22).

In the current study, with the aim of evaluating cnPCR in the identification of the etiological agent of VL in dogs, samples from the spleen, liver, kidneys and lymph nodes of 25 symptomatic (case group) and 15 asymptomatic animals were employed. The dogs were of different breeds, genders, and ages, and were from Teresina, Piauí state, Brazil. All case group animals and two controls tested positive in samples from the spleen, liver or lymph nodes by cnPCR. In symptomatic dogs, renal histopathological evaluation showed one animal (4\%) with amastigote forms of 
Leishmania in inflammatory infiltrate, while cnPCR in kidney samples detected Leishmania DNA in eight animals (32\%). In conclusion, there were more positive results by cnPCR in symptomatic seropositive dogs and cnPCR is more precise than conventional histopathology in detecting the Leishmania parasite (21).

In dogs from an endemic urban area in Paraná state, Brazil, of 169 studied animals, 11 (6.6\%) had anti-Leishmania braziliensis antibodies (titers $\geq$ 40) detected by immunofluorescent antibody test (IFAT) while four (2.4\%) showed L. braziliensis complex DNA by cnPCR. Such results indicate the importance of monitoring dogs to confirm the diagnosis of cutaneous leishmaniasis and also reveal the possibility of cross reactions in IFAT (24).

Others authors, evaluating cnPCR for diagnosis of VL in dogs from endemic areas (Poxoréo, MT, Brazil) using primers 13A and 13B for kDNA of Leishmania spp., obtained sensitivity of 55\%, specificity of $66.3 \%$ and a kappa coefficient of $0.2134(25,26)$. These results suggested that cnPCR is not an appropriate tool for screening in populations from endemic areas. Another study that compared the viability of blood samples from dogs naturally infected with VL in the south of Italy, obtained $85.3 \%$ of positive results by IFAT and $93.7 \%$ by cnPCR using primers $13 \mathrm{~A}$ and 13B (27). Despite the use of these two primers and DNA extraction by similar methods, the aforementioned studies showed different results, which suggests a low reproducibility of cnPCR for VL diagnosis in dog blood samples. Another possibility is that the efficiency of the technique is associated with the parasite load, that is, animals with a high parasite load would present positive results using the cnPCR technique.

According to some authors, the use of realtime PCR with primers derived from the RV1/ RV2 system in blood and bone marrow revealed an adequate correlation between parasite load and patient clinical status, which permit us to conclude that parasite quantification is necessary for an accurate reading of cnPCR results (28). Consequently, to check the progress of real-time PCR for VL diagnosis, researchers compared the cnPCR system (13A/13B) with qPCR (adapting the $13 \mathrm{~A} / 13 \mathrm{~B}$ system) in the bone marrow of dogs (29). They found 54 and $84 \%$ of positive results respectively for cnPCR and qPCR (29). The samples that tested negative for cnPCR showed a parasite load of less than 30 parasites/mL of bone marrow (29). Using peripheral blood samples from 15 dogs that were positive for VL, qPCR sensitivity was $100 \%$ (19). The system developed by these researchers elucidated the positive cases by cnPCR, evaluated the different responses to therapy and validated the use of blood collection as a less invasive method for diagnosis of VL.

We also obtained some promising results in the detection of L. infatum in naturally infected dogs by qPCR. Two animal groups were evaluated using a specific primer set developed for Leishmania donovani complex, in which a fragment of $132 \mathrm{bp}$ of kDNA from $L$. infantum was amplified (14). One group was composed of 21 dogs in which leishmaniasis clinical evidence was confirmed by parasitological examination or ELISA. The second group from non-endemic areas was negative in both assays. When canine blood samples were assessed using this system the limit of detection was found to be 0.07 parasite per reaction, the efficiency was $94.17 \%\left(\mathrm{R}^{2}=0.93\right.$, slope $=-3.47)$ and the sensitivity and specificity were respectively $100 \%$ and $83.33 \%$ (14).

Concerning cost-effectiveness, the operational advantages and data obtained in post-examination are satisfactory for institutions that use cnPCR as a diagnostic tool. In FIOCRUZ, Recife, Brazil, where there is a real-time PCR thermocycler and the technology is already implemented, the costs of cnPCR and qPCR become equivalent and similar to those described in Germany (30).

\section{FINAL COMMENTS}

qPCR is already available in several laboratories, especially in the private sector; however, the interpretation of its results requires new levels of knowledge. Therefore, it requires a trained staff to ensure the accuracy of the method (31). In addition to the operational advantages, qPCR is a sensitive and reproducible technique that could replace cnPCR in diagnostic routines. The possibility of deployment of qPCR in highly complex diagnoses in endemic areas of leishmaniasis would facilitate a swift and safe return for patients. The use of high sensitive technique that can monitor therapy and may prevent relapses promotes broader prospects for disease control.

Thereby, it is expected that the emergence of these new technologies will result in the 
development of new drugs and treatments, and particularly of diagnostic methods, and will lead to the improvement in the quality of life of patients (31). Given the above evidence, even some authors had previously defined cnPCR as "gold standard", qPCR will certainly be the technique of reference in the future (28).

\section{COPYRIGHT}

(c) CEVAP 2010

\section{SUBMISSION STATUS}

Received: June 22, 2010.

Accepted: August 19, 2010.

Abstract published online: August 23, 2010.

Full paper published online: November 30, 2010.

\section{CONFLICTS OF INTEREST}

There is no conflict.

\section{FINANCIAL SOURCE}

The National Council for Scientific and Technological Development $(\mathrm{CNPq})$ provided the financial support for M. Paiva-Cavalcanti's doctorate, which originated the present study.

\section{CORRESPONDENCE TO}

MILENA DE PAIVA-CAVALCANTI, Departamento de Imunologia, Centro de Pesquisas Aggeu Magalhães, FIOCRUZ, Av. Moraes Rego, s/n, Cidade Universitária, Recife, PE, 50.670-420, Brasil. Phone: +55 812101 2640. Fax: +55 81 21012679. Email: mp@cpqam. fiocruz.br.

\section{REFERENCES}

1. Chappuis F, Sundar S, Hailu A, Ghalib H, Rijal S, Peeling RW, et al. Visceral leishmaniasis: what are the needs for diagnosis, treatment and control? Nat Rev Microbiol. 2007;5(11):873-82.

2. Gomes YM, Paiva-Cavalcanti M, Lira RA, Abath FG, Alves LC. Diagnosis of canine visceral leishmaniasis: biotechnological advances. Vet J. 2008;175(1):45-52.

3. Schallig HD, Oskam L. Molecular biological applications in the diagnosis and control of leishmaniasis and parasite identification. Trop Med Int Health. 2002;7(8): 641-51.

4. Nicolas L, Prina E, Lang T, Milon G. Realtime PCR for detection and quantitation of Leishmania in mouse tissues. J Clin Microbiol. 2002;40(5):1666-9.

5. Zarlenga DS, Higgins J. PCR as a diagnostic and quantitative technique in veterinary parasitology. Vet Parasitol. 2001;101(3-4):215-30.

6. Bastien P, Procop GW, Reischl U. Quantitative realtime PCR is not more sensitive than conventional PCR. J Clin Microbiol. 2008;46(6):1897-900.

7. Yang S, Rothman RE. PCR-based diagnostics for infectious diseases: uses, limitations and future applications in acute-care settings. Lancet Infect Dis. 2004;4(6):337-48.

8. Bahk YY, Kim SA, Kim JS, Euh HJ, Bai GH, Cho $\mathrm{SN}$, et al. Antigens secreted from Mycobacterium tuberculosis: identification by proteomics approach and test for diagnostic marker. Proteomics. 2004;4(11):3299-307.

9. Higuchi R, Dollinger G, Walsh PS, Griffith R. Simultaneous amplification and detection of specific DNA sequences. Biotechnology. 1992;10(4):413-7.

10. Mortarino M, Franceschi A, Mancianti F, Bazzocchi C, Genchi C, Bandi C. Quantitative PCR in the diagnosis of Leishmania. Parassitologia. 2004;46(1-2):163-7.

11. Kubista M, Andrade JM, Bengtsson M, Forootan A, Jonák J, et al. The real time polymerase chain reaction. Mol Aspects Med. 2006;27(2-3):95-125.

12. Applied Biosystems. Real Time PCR Systems. Absolute Quantification Getting Started Guide. Atlanta, 2005. Available from: http://www. appliedbiosystems.com. Access in: Aug. 16, 2006.

13. Too HP. Real time PCR quantification of GFR2 alternatively spliced isoforms in murine brain and peripheral tissues. Mol Brain Res. 2003;114(2):146-53.

14. Paiva-Cavalcanti M, Brito MEF, Souza WV, Gomes YM, Abath FGC. The development of a real-time PCR assay for the quantification of Leishmania infantum DNA in canine blood. Vet J. 2009;182(2):356-8.

15. Ferreira ID, Rosário VE, Cravo PV. Real-time quantitative PCR with SYBR Green I detection for estimating copy numbers of nine drug resistance candidate genes in Plasmodium falciparum. Malar J. 2006;5:1

16. Robledo G, González CI, Morillo C, Martín J, González A. Association study of PTPN22 C1858T polymorphism in Trypanosoma cruzi infection. Tissue Antigens. 2007;69(3):261-4.

17. Jonhson VJ, Yucesoy YB, Luster MI. Genotyping of single nucleotide polymorphisms in cytokine genes using real-time PCR allelic discrimination technology. Cytokine. 2004;27(6):135-41.

18. Mullis KB, Faloona FA. Specific synthesis of DNA in vitro via a polimerase-catalyzed reaction. Methods Enzymol. 1987;155:335-50.

19. Fichoux YL, Quaranta JF, Aufeuvre JP, Lelievre A, Marty P, Suffia I, Rousseau D, et al. Occurrence 
of Leishmania infantum parasitemia in asymptomatic blood donors living in an area of endemicity in southern France. J Clin Microbiol. 1999;37(6):1953-7.

20. Ravel S, Cuny G, Reynes J, Veas F. A highly sensitive and rapid procedure for direct PCR detection of Leishmania infantum within human peripheral blood mononuclear cells. Acta Trop. 1995;59(3):187-96.

21. Soares MJV, Noraes JRE, Roselino AMF. Polymerase chain reaction in detecting Leishmania sp. symptomatic and asymptomatic seropositive dogs. J Venom Anim Toxins incl Trop Dis. 2005;11(4):532-9.

22. Lachaud L, Chabbert E, Dubessay P, Dereure J, Lamothe J, Dedet JP, et al. Value of two PCR methods for the diagnosis of canine visceral leishmaniasis and the detection of asymptomatic carriers. Parasitology. 2002;125(Pt 3):197-207.

23. Gomes AHS, Ferreira IMR, Lima MLSR, Cunha EA, Garcia AS, Araújo MFL, et al. PCR identification of Leishmania in diagnosis and control of canine leishmaniasis. Vet Parasitol. 2007;144(3-4):234-41.

24. Cerino DA, Veloso J, Perles TF, Zanzarini PD, Lonardoni MVC, Silveira TGV. American cutaneous leishmaniasis in dogs from an endemic urban area in Cianorte municipality, Paraná State, Brazil. J Venom Anim Toxins incl Trop Dis. 2010;16(2): 241-52.

25. Rodgers MR, Popper SJ, Wirth DF. Amplification of Kinetoplast DNA as a tool in the detection and diagnosis of Leishmania. Exp Parasitol. 1990;71(3):267-75.
26. Nunes CM, Dias AKK, Gottardi FP, Paula HB, Azevedo MAA, Lima VMF. Avaliação da reação em cadeia da polimerase para diagnóstico da leishmaniose visceral em sangue de cães. Rev Bras Parasitol Vet. 2007;16(1):5-9.

27. Manna L, Vitale F, Reale S, Caracappa S, Pavone LM, Morte RD, et al. Comparison of different tisuue sampling for PCR-based diagnosis and follow-up of canine visceral leishmaniosis. Vet Parasitol. 2004;125(3-4):251-62.

28. Mary C, Faraut F, Lascombe L, Dumon H. Quantification of Leishmania infantum DNA by a real-time PCR assay with high sensitivity. J Clin Microbiol. 2004;42(11): 5249-55.

29. Francino O, Altet L, Sanchez-Robert E, Rodriguez A, Solano-Gallego L, Alberola J, et al. Advantages of real-time PCR assay for diagnosis and monitoring of canine leishmaniosis. Vet Parasitol. 2006;137(3-4):214-21.

30. Schulz A, Mellenthin K, Schonian G, Fleischer B, Drosten C. Detection, differentiation, and quantitation of pathogenic Leishmania organisms by a fluorescence resonance energy transferbased real-time PCR assay. J Clin Microbiol. 2003;41(4):1529-35.

31. Paiva-Cavalcanti M, Lorena VMB, Gomes YM. Avanços biotecnológicos para o diagnóstico das doenças infecciosas e parasitárias. Rev Patol Trop. 2008;37(1):1-14. 